\title{
DETERMINANTES DIETÉTICOS DEL CONSUMO DE ZINC EN MENORES DE CINCO AÑOS CON RETARDO DEL CRECIMIENTO EN COMUNIDADES MAYAS DE GUATEMALA
}

\author{
Michele Monroy-Valle ${ }^{1,2 a, d}$, Wendy Coyoy²,a , Jorge De León ${ }^{3, b, e}$, Iván D. Flórez,,,f
}

\begin{abstract}
RESUMEN
El objetivo del estudio fue identificar elementos del patrón alimentario que influyen en la ingesta y biodisponibilidad de zinc en niños con retardo del crecimiento (RC) entre 1-5 años en comunidades maya de la localidad de Huehuetenango, Guatemala. Estudio descriptivo, transversal aplicado en 138 niños de entre 1-5 años con RC. Se realizó un inventario de disponibilidad alimentaria de fuentes de zinc, se construyó un cuestionario frecuencia de consumo de alimentos, un cuestionario de condiciones de vida, prácticas alimentarias, antropometría, y se recolectó información acerca de enfermedades infecciosas. Hubo disponibilidad de 28 alimentos fuentes de zinc $(54 \%$ de baja y $7 \%$ de alta biodisponibilidad). El consumo de alimentos de alta biodisponibilidad fue bajo y el de alimentos antagonistas, alto. Los niños consumían $3 \mathrm{mg}$ de zinc al día (IC95\% 2,65-3,35). Solo el 14,5\% alcanzaba los requerimientos de zinc, y el 2,9\% alcanzaba la recomendación diaria. La ocurrencia de diarrea e infecciones, en los quince días previos, fue del 29,71 y $45,65 \%$, respectivamente. Concluimos que la mayoría de niños con RC tuvo un bajo consumo de fuentes de zinc, alto consumo de alimentos antagonistas.
\end{abstract}

Palabras clave: Zinc; Deficiencia de zinc; Desnutrición; Insuficiencia de crecimiento; Consumo de alimentos (fuente: DeCS BIREME).

\section{DIETETIC DETERMINANTS OF ZINC CONSUMPTION IN STUNTED CHILDREN UNDER FIVE IN MAYA COMMUNITIES FROM GUATEMALA}

\begin{abstract}
The objective of the study was to identify the elements from feeding patterns that influence the intake and bioavailability of zinc in stunted children (SC) 1-5 years from Maya communities living in Huehuetenango, Guatemala. This descriptive, cross-sectional study was performed in 138 stunted children aged 1-5 years. It was applied: an inventory of zinc food sources availability, a Food Frequency Questionnaire, a questionnaire about living conditions. anthropometrics measurements and information on food intake habits, and information about disease prevalence. Twenty-eight food sources of zinc were available for consumption ( $54 \%$ low and $7 \%$ high zinc bioavailability). The consumption of foods with high bioavailability was low, while the antagonistic foods were high. On average the daily zinc consumption in diet $3 \mathrm{mg}$ (95\% Cl: $2.65-3.35)$. Only $14.5 \%$ of the children met the zinc requirements, and $2.9 \%$ reached the daily recommendation. Episodes of diarrhea and respiratory infections were observed 15 days before the visit, in $29.71 \%$ and $45.6 \%$ of children, respectively. Most of children have a diet that does not cover the daily requirement.
\end{abstract}

Key words: Zinc; Zinc deficiency; Malnutrition; Failure to thrive; malnutrition, Food consumption (source: MeSH NLM).

\section{INTRODUCCIÓN}

El zinc desempeña un rol fundamental en el crecimiento y el desarrollo infantil, por ser cofactor de aproximadamente 300 enzimas en el recambio celular ${ }^{(1)}$. Su deficiencia se asocia con la desaceleración del crecimiento, déficit inmune, alteraciones del tracto gastrointestinal, aumento del estrés oxidativo y generación de estados proinflamatorios, trayendo como consecuencia el retardo del crecimiento $(\mathrm{RC})$ y la predisposición a infecciones ${ }^{(2,3)}$.

\footnotetext{
1 Unidad de Investigación en Seguridad Alimentaria y Nutricional, Instituto de Investigaciones Químicas y Biológicas. Universidad de San Carlos de Guatemala, Guatemala de la Asunción, Guatemala.

2 Facultad de Ciencias de la Salud, Universidad Rafael Landívar. Guatemala de la Asunción, Guatemala.

3 Unidad de Bioestadística. Facultad de Ciencias Químicas y Farmacia. Universidad de San Carlos de Guatemala. Guatemala de la Asunción, Guatemala.

Department of Health Research Methods, Evidence and Impact, McMaster University. Hamilton, Canadá.

a Nutricionista, ${ }^{\mathrm{b}}$ químico biólogo, ${ }^{\mathrm{c}}$ médico pediatra, ${ }^{\mathrm{d}}$ magíster en Epidemiología y Salud Pública, ${ }^{\mathrm{e}} \mathrm{PhD}$ en Salud Pública, ${ }^{\mathrm{f}}$ magíster en Epidemiología Clínica Recibido: 21/07/2016 Aprobado: 09/08/2017 En línea: 29/09/2017
}

Citar como: Monroy-Valle M, Coyoy W, De León J, Flórez ID. Determinantes dietéticos del consumo de zinc en menores de cinco años con retardo de crecimiento en comunidades mayas de Guatemala. Rev Peru Med Exp Salud Publica. 2017;34(3):451-8. doi:10.17843/rpmesp.2017.343.2276 
La deficiencia de zinc es un problema de salud pública cuando su prevalencia supera el $20 \%{ }^{\text {(3) }}$. En Guatemala, esta alcanza $34,9 \%$ e, incluso, $46,7 \%$ en algunas regiones ${ }^{(4)}$, zonas que también presentan prevalencias elevadas de RC ${ }^{(5)}$. Con base en el indicador "riesgo de deficiencia de zinc" propuesto por Wessells ${ }^{(6)}$, el cual integra la prevalencia estimada de consumo inadecuado de zinc y la de RC, Guatemala es uno de los países con mayor riesgo de deficiencia en Latinoamérica ${ }^{(7)}$.

La deficiencia de zinc es habitualmente más alta entre los grupos desfavorecidos y vulnerables, con menos acceso a servicios de salud, como las poblaciones indígenas ${ }^{(7,8)}$. La evidencia disponible sobre el impacto de la suplementación preventiva de zinc respalda la necesidad implementar intervenciones para aumentar la ingesta de este micronutriente en dichas poblaciones ${ }^{\left({ }^{(8)}\right.}$.

En países en desarrollo, las principales fuentes de zinc son vegetales (raíces, tubérculos, hortalizas, arroz, maíz, cereales), los cuales tienen alta relación molar fitato:zinc $(15: 1)$ lo que se traduce en una baja biodisponibilidad (2). Intervenciones dirigidas a este problema serían de gran impacto en mejorar la disponibilidad del zinc. Por ejemplo, un estudio desarrollado en Guatemala en niños cuyo alimento básico era el maíz, logró un aumento en la absorción de zinc usando un tipo de maíz bajo en fitatos ${ }^{(9)}$. Las poblaciones vulnerables suelen consumir poca cantidad de alimentos ricos en zinc y tener elevada ingesta de alimentos con altos fitatos (frijol) que disminuyen significativamente su biodisponibilidad (10). No obstante, se desconoce el tipo y cantidad de fuentes alimentarias de zinc en la dieta básica de Guatemala.

Este estudio buscó cuantificar el consumo de zinc dietario en niños de uno a cinco años con RC en cuatro localidades de la región del noroccidente de Guatemala con población indígena y evaluar la biodisponibilidad dietética del zinc.

\section{EL ESTUDIO}

Estudio de tipo transversal desarrollado en 2014 en cuatro municipios de Huehuetenango, departamento con mayor prevalencia de RC en el país ${ }^{(11)}$. Los municipios elegidos, están poblados por las etnias mam y chuj, pertenecientes a los mayas. La prevalencia de pobreza es alta: La Libertad $75 \%$, La Democracia 71\%, San Mateo Ixtatán 77\% y Chiantla $65 \%{ }^{(12)}$.

Se consideró el riesgo de deficiencia de zinc para el cálculo de la muestra. Para 2014, Huehuetenango tenía 204786 hogares, cerca de $45 \%$ con al menos un niño menor de cinco años (91 800 hogares) ${ }^{(13)}$ y de ellos $70 \%$ con RC (11) (64 260 niños). Entendiendo que cada hogar podía aportar entre uno a dos niños (poco frecuente más de tres

\section{MENSAJES CLAVE}

Motivación para realizar el estudio. Es importante cuantificar el consumo de zinc dietario en niños con retardo en el crecimiento, para identificar brechas de ingesta que sugieran recomendaciones adaptadas a los recursos

Principales hallazgos. El consumo medio de zinc fue de 2,65 $\mathrm{mg} /$ día en niños de $1-3$ y de $3.5 \mathrm{mg} / \mathrm{día}$ en niños de 3-5. El 85\% de la muestra no consume suficiente zinc dietario, las fuentes alimentarias son de baja biodisponibilidad y los niños no reciben la suplementación recomendada para su condición.

Implicancias. Garantizar la suplementación de zinc oral es necesaria ya que la ingesta dietética de la mayoría de la muestra no cubrió los requerimientos diarios para el Retardo en el Crecimiento.

niños/hogar en el rango de edad que tuvieran 1-4 años), los cálculos se realizaron buscando identificar el número ideal de niños, no de hogares, para completar la muestra. Se calculó la muestra con el programa EpiDat 4,0, teniendo en cuenta una deficiencia esperada de $90 \%$ en niños con RC ${ }^{(14)}$, nivel de confianza de 95\%, y una población esperada de 64260 niños, con precisión 5\%. La muestra resultante fue 185 niños. Se reclutaron los niños en los hogares hasta que se lograra completar el número. En caso que no se encontrara el niño en la casa, se le excluía y se reemplazaba con el siguiente niño que cumpliera criterios.

La Dirección de Área de Salud de Huehuetenango brindó información de los casos de niños con $\mathrm{RC}$ de cada municipio. A partir de la lista, se visitaron los hogares en el orden de la lista y que geográficamente fuera más conveniente de acceder, por continuidad entre los hogares, hasta completar la muestra. A cada madre se le solicitó un consentimiento informado y verbal sobre el estudio. Todos los niños incluidos tenían RC moderado o severo.

La definición de RC se basó en la establecida por el patrón de la OMS: RC moderado, entre -2 y -3 desviaciones estándar (DE) de talla para la edad (T/E); y RC severo, menor de -3 DE de T/E, que pertenecieran a alguna etnia maya (mam o chuj), residentes en municipios de estudio. Se excluyeron niños cuyas madres no consintieron participar, con malformaciones genéticas, y aquellos que no estuvieran presentes durante la recolección de datos. Los casos encontrados sin suplementación fueron reportados al Ministerio de Salud para que le dieran seguimiento. Para comunicarse con la madre se usó intérpretes para idiomas chuj y mam.

\section{VARIABLES}

Las variables estudiadas fueron: fuentes alimentarias de zinc, gramos de zinc y proteína, prácticas culinarias y 
alimentarias; dieta mixta (baja, mediay alta biodisponibilidad de zinc); presencia de infecciones respiratorias y diarrea en 15 días previos.

\section{PROCEDIMIENTOS}

Se recolectaron datos en dos etapas. Primero, a partir de los vendedores locales, se realizó un inventario de existencia local de alimentos para identificar los alimentos disponibles en tiendas y/o mercados, y su precio (15). Para la determinación de alimentos fuentes se utilizó el Reglamento Técnico Centroamericano ${ }^{(16)}$, donde establece que un alimento fuente de zinc contiene como valor de referencia del nutriente (VRN), no menos de $15 \% / 100 \mathrm{~g}$ (alimento sólido), 7,5\%/100 mL (alimento líquido) o 10\%/ porción de alimento. La determinación del VRN se basó en el promedio de ingesta recomendada de zinc en $\mathrm{mg} /$ día para la edad según valores de referencia de ingesta de proteínas y zinc del comité conjunto FAO/OMS/UNU para niños de 1 a 5 años ${ }^{(1,17)}$.

Para la clasificación de los alimentos fuentes de zinc, e inhibidores de absorción (fitatos) se utilizó la Tabla de Composición Química de Alimentos, de USDA (18) y la Tabla de Composición de Alimentos del INCAP (TCA) ${ }^{(19) .}$ Se construyó un listado de 72 alimentos para descartar de acuerdo a disponibilidad local. Para cuantificar el consumo diario de zinc los suplementos fueron excluidos.

La segunda etapa fue la recolección de la información de consumo y prácticas alimentarias y dietéticas. Se elaboró una frecuencia de consumo de alimentos (FCA) (20) semicuantitativa tipo tamizaje de block, con base en el listado de alimentos del inventario local (21), de la cual se obtuvo la cantidad de gramos de zinc y proteína consumidos. Se recolectó información sobre condiciones socioeconómicas, antropometría, e infecciones respiratorias y episodios diarreicos 15 días y 6 meses previos a la inclusión. Se evaluaron prácticas culinarias y alimentarias en el hogar, como la nixtamalización de maíz (cocción en agua con $\mathrm{pH}$ básico usando carbonato de calcio), remojo del frijol, preparación del frijol después de cocido, cocción de vegetales de hoja verde, consumo de tortilla y café. Se conoce que estas prácticas pueden reducir la cantidad de fitatos y mejorar la biodisponibilidad de zinc ${ }^{(22,23)}$.

El análisis se hizo por subgrupos de población según rangos de edad 1-3 y 3-5 años. Se usó medidas de resumen de acuerdo al tipo de variables (media y DE para cuantitativas, frecuencias y proporciones para cualitativas). Se utilizó el programa Microsoft Office Excel 2007.

El protocolo fue aprobado por el Comité de Ética e Investigación de Facultad de Ciencias de la Salud, Universidad Rafael Landívar, Ciudad de Guatemala.

\section{RESULTADOS}

Se incluyeron 185 niños, en 25 casos las madres rechazaran su participación. Además, 22 no se encontraron al momento de la visita. Se incluyeron finalmente 138 niños ( 85 hogares) en el análisis. Hubo RC moderado en 59 niños $(42,8 \%)$ y severo en $79(57,2 \%)$. El RC severo fue más frecuente en niños de $1-3$ años $(69,1 \%)$, y el moderado más frecuente en niños 3-5 años $(59,6 \%)$.

De los 72 alimentos posibles fuentes de zinc, 28 estaban disponibles en los municipios y $54 \%(n=15)$ de ellos son de baja, 39\% $(n=11)$ de moderada y $7 \%(n=2)$ de alta biodisponibilidad de zinc. Los alimentos considerados muy caros por los vendedores fueron: cereal de desayuno fortificado, atún enlatado, pan sándwich blanco, leche líquida, queso fresco, jamón y carne de res fresca. Los alimentos considerados "de buen precio" fueron frijol negro, arroz, güisquil o chayote, quilete (Solanum americanum, vegetal autóctono de hoja verde), cereal de corazón de trigo, salchicha de res o cerdo y vísceras de res (hígado, lengua, riñón y corazón).

Los alimentos de baja biodisponibilidad, consumidos de 1-2 veces por semana, fueron: frijol negro $(70 \%)$, arroz $(80 \%)$, pasta $(67 \%)$, aguacate $(48 \%)$, güisquil/chayote $(46 \%)$, brócoli (36\%); de moderada biodisponibilidad: huevos $(62 \%)$, pollo (52\%), salchicha de res/cerdo $(24 \%)$, leche de vaca en polvo (10\%), queso fresco/de capas $(8 \%$ ) y gallina (7\%); y con alta biodisponibilidad: carne de res $(31 \%)$, y vísceras (8\%). El quilete, de baja biodisponibilidad, fue el más consumido (41\%), 3-4 veces por semana. El consumo de inhibidores de absorción de zinc fue cinco o más veces por semana: tortilla de maíz ( $99 \%)$, bebida de maíz o atol $(40 \%)$. El patrón de consumo según biodisponibilidad fue menos de una vez semanal (alimentos de alta), dos veces (alimentos de moderada) y cuatro veces por semana (alimentos de baja), y diario para inhibidores de absorción del zinc (Tabla 1).

De acuerdo a la clasificación de biodisponibilidad de zinc en dietas mixtas ${ }^{(6)}, 38 \%, 10 \%$ y $52 \%$ de dietas fueron consideradas de alta, moderada y baja disponibilidad, respectivamente. Sin embargo, debido al consumo de alimentos con alto contenido de proteína vegetal, fibra, y fitatos, y bajo consumo de proteína de origen animal y de fuentes alimentarias de zinc, todas las dietas serian consideradas de baja biodisponibilidad (Tabla 1).

Las prácticas culinarias más importantes fueron: nixtamalización de maíz (realizada en todos los hogares para preparar tortillas) y, de ellos, el $61,2 \%$ ( $n=52)$ la realizó adecuadamente, el remojo del frijol durante más de ocho horas únicamente lo realizó el 5,9\% $(n=5)$ y la preparación del frijol después de cocido $43,5 \%$ ( $n=37$ ) lo realiza, los 
Tabla 1. Clasificación de biodisponibilidad de zinc en dietas mixtas de niños de 1 a 5 años. Huehuetenango, Guatemala. 2014

\begin{tabular}{|c|c|c|c|c|}
\hline $\begin{array}{l}\text { Clasificación } \\
\text { Biodisponibilidad }\end{array}$ & Fuente de zinc & Criterios de la dieta mixta & $\begin{array}{l}\% \text { dietas* en } \\
\text { el patrón de } \\
\text { consumo }\end{array}$ & $\begin{array}{l}\% \text { dietas }^{\dagger} \text { en } \\
\text { el patrón de } \\
\text { consumo }\end{array}$ \\
\hline Alta & $\begin{array}{l}\text { Carne de res, vísceras } \\
\text { (hígado, lengua, riñón, } \\
\text { corazón) }\end{array}$ & $\begin{array}{l}\text { 1. Consumo bajo en fibra. } \\
\text { 2. Consumo semanal de alimentos fuentes de } \\
\text { fitato (frijol, tortilla, arroz, y quilete [Solanum } \\
\text { americanum]). } \\
\text { 3. Consumo de carne de res, vísceras o pollo, } \\
\text { 3-4 veces a la semana. } \\
\text { 4. Consumo de tres de los siguientes } \\
\text { alimentos por lo menos una vez a la semana: } \\
\text { pollo, gallina, cerdo, jamón, salchicha, crema, } \\
\text { queso, leche, huevos, atún en agua. }\end{array}$ & 38 & 0 \\
\hline Moderada & $\begin{array}{c}\text { Pollo, gallina, cerdo, jamón, } \\
\text { salchicha, crema, queso, } \\
\text { leche, huevos, atún en } \\
\text { agua. }\end{array}$ & $\begin{array}{l}\text { 1. Consumo de alguno de los siguientes } \\
\text { alimentos cinco o más veces a la semana: } \\
\text { gallina, cerdo, jamón, salchicha, crema, } \\
\text { queso, leche, huevos, atún en agua, } \\
\text { 2. Consumo tres de los siguientes alimentos } \\
\text { por lo menos una vez a la semana; gallina, } \\
\text { cerdo, jamón, salchicha, crema, queso, } \\
\text { leche, huevos, atún en agua. }\end{array}$ & 10 & 0 \\
\hline Baja & $\begin{array}{l}\text { Frijol, arroz, incaparina, } \\
\text { manías, aguacate, brócoli, } \\
\text { güisquil, quilete, avena } \\
\text { en hojuelas, cereal de } \\
\text { desayuno, harina de arroz, } \\
\text { harina de maíz, pasta, } \\
\text { corazón de trigo, pan } \\
\text { sándwich. }\end{array}$ & $\begin{array}{l}\text { 1. Consumo diario de alimentos fuentes de } \\
\text { fitato: arroz, frijol, quilete. } \\
\text { 2. Consumo diario de alimentos fuente de } \\
\text { fibra. }\end{array}$ & 52 & 100 \\
\hline $\begin{array}{l}\text { Inhibidores de } \\
\text { absorción }\end{array}$ & $\begin{array}{l}\text { Tortilla de maíz, bebida de } \\
\text { maíz y pan dulce. }\end{array}$ & $\begin{array}{l}\text { 1. Consumo diario de alimentos fuentes de } \\
\text { fitato. }\end{array}$ & & \\
\hline
\end{tabular}

* Tomando en cuenta únicamente el aporte de proteína

† Tomando en cuenta la dieta en su totalidad (patrón alimentario)

vegetales de hoja verde $92,9 \%(n=79)$ los cuecen. En las prácticas alimentarias el $99,3 \%(n=137)$ de niños consumía tortilla diariamente (media: 5 unidades al día), 93\% $(n=172)$ consumen café diariamente (media: 2 tazas al día).

En la FCA se identificó que 14,6\% ( $n=27)$ de niños consumen el requerimiento diario de zinc. El consumo medio de zinc fue $2,65 \mathrm{mg} / \mathrm{día}$ (DE: 2,04 ) y $3,5 \mathrm{mg} / \mathrm{día}(\mathrm{DE}: 2,15$ ) en niños de 1-3 y 3-5 años, respectivamente. El consumo medio de proteína fue $17,25 \mathrm{~g}$ (DE: 9,77) y 23,4 g (DE 12,1) en niños de 1-3 y 3-5 años, respectivamente (Tabla 2 ). Los niños de 1-3 años deberían consumir 4,1 mg/día de zinc, y los de 3-5 años, 3,5 mg/día (Figura 1).

En seis meses anteriores al estudio, 22 niños $(27,2 \%)$ de 1-3 años y 29 niños (50,9\%) de 3-5 años, habían recibido tabletas de zinc (20 mg/día) o micronutrientes espolvoreados (gluconato de zinc $4,1 \mathrm{mg} / \mathrm{sobre}$ ). Sin embargo, en el momento de la entrevista ninguno recibía la suplementación.
Al momento de la entrevista 41 niños $(29,7 \%)$ habían tenido diarrea en los 15 días previos, episodio que fue corto (3-4 días) en la mayoría de casos (82,9\%). Ochenta niños $(57,97 \%)$ tuvieron al menos un episodio de diarrea en últimos seis meses. La media de episodios diarreicos en los últimos seis meses fue 1,6 (DE: 1,78). Habían tenido infección respiratoria durante 15 días previos, 63 niños (45,7\%), y la media fue 1,04 episodios (DE: 0,21). Los episodios fueron de 3-4 días en 90 casos (65,2\%). Durante los últimos seis meses tuvieron al menos una infección respiratoria 93 niños $(67,4 \%)$, y la media de episodios fue 2,35 (DE: 1,31 ).

\section{DISCUSIÓN}

En el presente estudio describimos cómo en la población de niños entre 1-5 años de una zona indígena del noroccidente de Guatemala, la alimentación fue, en la totalidad de los casos, considerada de baja disponibilidad de zinc y la mayoría de los niños consumían menos zinc del requerido diariamente dada su condición de RC. 
Tabla 2. Ingesta de zinc y proteína en dietas mixtas de menores de 5 años. Huehuetenango, Guatemala

\begin{tabular}{|c|c|c|c|c|}
\hline \multirow[t]{2}{*}{ Característica } & $\begin{array}{l}\text { Niños que no consumen } \\
\text { la recomendación (RNI)* }\end{array}$ & $\begin{array}{c}\text { Niños que no consumen el } \\
\text { requerimiento }(E A R)^{\dagger}\end{array}$ & Ingesta (mg/día) & \multirow[t]{2}{*}{$\mathbf{n}$} \\
\hline & $\mathrm{n}(\%)$ & $\mathrm{n}(\%)$ & Media (DE) & \\
\hline \multicolumn{5}{|l|}{ Zinc } \\
\hline \multicolumn{5}{|l|}{ Edad (años) } \\
\hline $1-3$ & $79(97,5)$ & $71(87,7)$ & $2.65(2,04)$ & 81 \\
\hline $3-5$ & $55(96,5)$ & $47(82,5)$ & $3.5(2,15)$ & 57 \\
\hline Total & $134(97,5)$ & $118(85,5)$ & $3(2,12)$ & 138 \\
\hline \multicolumn{5}{|l|}{ Sexo } \\
\hline Masculino & $72(96,0)$ & $63(84,0)$ & $3.23(2,31)$ & 75 \\
\hline Femenino & $62(98,4)$ & $55(87,3)$ & $2.73(1,86)$ & 63 \\
\hline \multicolumn{5}{|l|}{ Proteína† } \\
\hline \multicolumn{5}{|l|}{ Edad (años) } \\
\hline $1-3$ & -- & $15(18,5)$ & $17.2(9,77)$ & 81 \\
\hline $3-5$ & -- & $9(15,8)$ & $23.4(12,1)$ & 57 \\
\hline \multicolumn{5}{|l|}{ Sexo } \\
\hline Masculino & -- & $12(16,0)$ & $20.9(11,5)$ & 75 \\
\hline Femenino & -- & $12(19,0)$ & $18.4(10,6)$ & 63 \\
\hline
\end{tabular}

* Se utilizó el requerimiento y la recomendación de zinc según la edad para una dieta de baja biodisponibilidad de zinc, este dato se comparó con la ingesta en mg de zinc consumidos un día antes.

† Se determinó a partir de los gramos de proteína consumidos en promedio una semana previa al estudio comparando con el requerimiento de proteína en $\mathrm{g} / \mathrm{kg}$ al día.

RNI: Reference Nutrient Intakes; EAR: Estimated Average requirements; DE: desviación estándar.

Existen pocos estudios sobre el consumo dietético de zinc, sin embargo, un estudio desarrollado en las áreas rurales de la región autónoma del Tíbet encontró, igual que el presente estudio, que la biodisponibilidad de $\mathrm{Ca}, \mathrm{Fe}$ y $\mathrm{Zn}$ puede ser motivo de preocupación debido al alto contenido de ácido fítico en la dieta de los niños pequeños ${ }^{(24)}$.
El alto consumo de alimentos de baja biodisponibilidad, aunado al alto consumo de inhibidores de la absorción y utilización del zinc en el organismo, junto con las prácticas culinarias ${ }^{(22)}$ y alimentarias inadecuadas para disminuir la cantidad de fitatos en los alimentos (23), pudiesen explicar la baja ingesta de zinc encontrada.

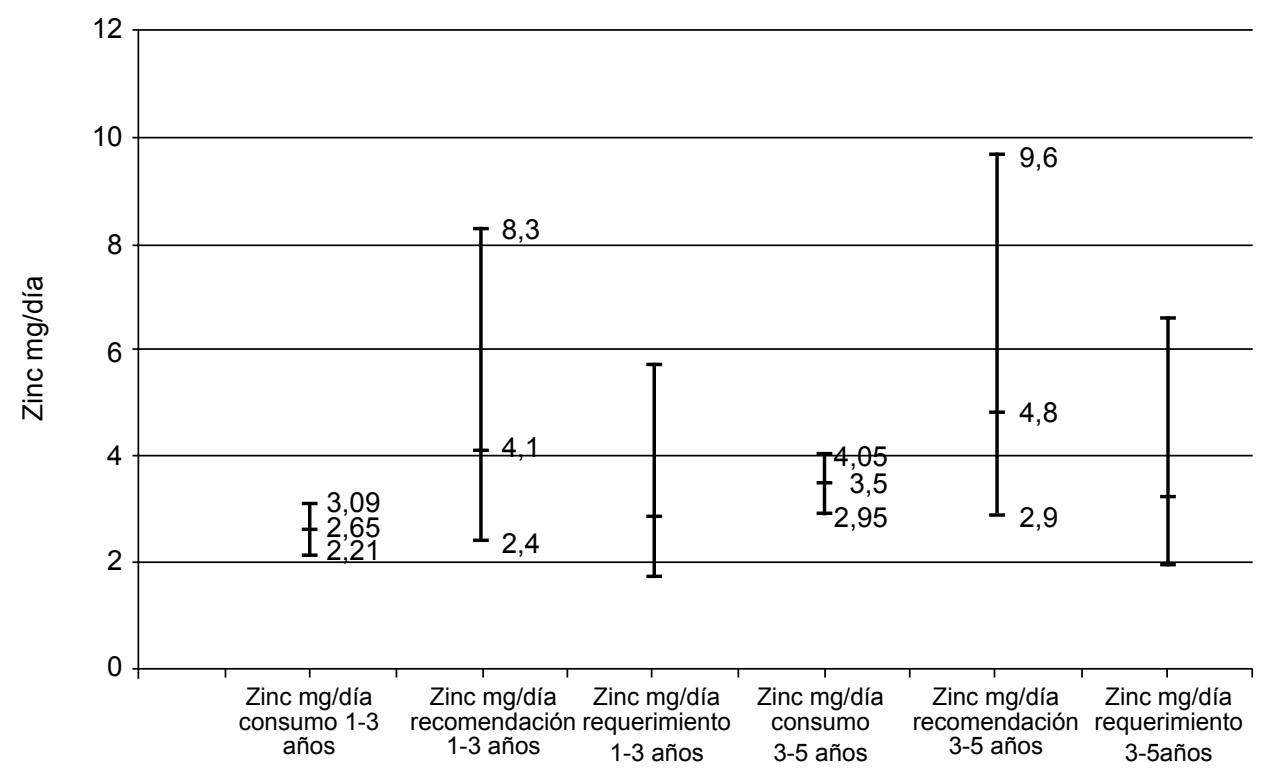

Nota: Los valores de la recomendación y el requerimiento están basados en el peso promedio según la edad de un niño sin desnutrición conforme a los patrones de crecimiento de OMS. El rango inferior de requerimiento y recomendación representan la cantidad en mg/día que debiera consumir un niño con dieta de alta biodisponibilidad de zinc y el rango superior es la cantidad recomendada para dietas de baja biodisponibilidad de zinc.

Figura 1. Consumo de Zinc (mg/día) comparados con la recomendación en alta, moderada y baja biodisponibilidad en la dieta. Huehuetenango, Guatemala 
Tabla 3. Porcentaje de niños de 1 a 5 años de edad, clasificados con retardo en crecimiento (RC) según características seleccionadas

\begin{tabular}{|c|c|c|c|}
\hline \multirow{2}{*}{ Característica } & \multicolumn{2}{|c|}{$\%$ de niños con RC (T/E) } & \multirow{2}{*}{$\mathbf{n}$} \\
\hline & Severa* $^{*}$ & Moderada $^{\dagger}$ & \\
\hline \multicolumn{4}{|l|}{ Edad (años) } \\
\hline $1-3$ & 69,1 & 30,9 & 81 \\
\hline $3-5$ & 40,4 & 59,6 & 57 \\
\hline \multicolumn{4}{|l|}{ Sexo } \\
\hline Masculino & 60,0 & 40,0 & 75 \\
\hline Femenino & 54,0 & 46,0 & 63 \\
\hline Total & 57,2 & 42,8 & 138 \\
\hline
\end{tabular}

Cada índice se expresa en términos del número de desviaciones estándar (DE) de la media del patrón de referencia internacional utilizado por OMS.

* Niños y niñas que están < -3 DE por debajo de la media.

† Niños y niñas que están < -2 Desviaciones estándar a -3DE.

T/E: talla para la edad.

Si bien, en las comunidades existen alimentos fuente de zinc y de proteína animal, muchos fueron considerados de alto costo, lo que disminuye la disponibilidad en el hogar, predominando aquellos de baja disponibilidad y bajo costo. En Huehuetenango se cultiva principalmente maíz y frijol (25) y, por ser de autoproducción, hay mayor disponibilidad en el hogar, mientras que los alimentos con alta biodisponibilidad deben ser adquiridos, además que su distribución en el mercado puede estar limitada al acceso, vías de transporte y a los altos índices de pobreza en esta región ${ }^{(12)}$.

A pesar de que en algunos casos se alcanza el requerimiento de zinc, se debe considerar que el contenido total en la dieta solo provee un cálculo teórico que es absorbido y utilizado por los tejidos corporales ${ }^{(26)}$. El alto consumo de alimentos ricos en fitatos en esta población fue mayor que el de alimentos fuente de zinc. Los requerimientos de zinc de los niños con RC se estiman entre $2 \mathrm{mg} / \mathrm{kg}$ y $4 \mathrm{mg} / \mathrm{kg}$ de peso corporal ${ }^{27)}$, los cuales son más altos que los de los niños sanos debido al agotamiento previo del zinc y la reducción de la absorción por los cambios en el tracto intestinal ${ }^{(28)}$.

Entre los niños de 1-3 años, el 18,5\% no alcanza el requerimiento de proteína, y el $15,8 \%$ no lo alcanza en la población de 3-5 años. Además, el aporte proteico proviene, principalmente, de alimentos de origen vegetal de baja calidad. Debido la potenciación que la proteína hace en la absorción y utilización de zinc, la baja proteína, y de baja calidad, empeora el escenario de la deficiencia de zinc ${ }^{(29)}$.

La deficiencia de zinc se relaciona con mayor número de infecciones, e infecciones recurrentes se asocian con inapetencia y menor ingesta de alimentos, poca ganancia de peso, y más deficiencias de micronutrientes ${ }^{(30,31)}$. Las infecciones gastrointestinales y respiratorias frecuentes llevan a aumento en los requerimientos de zinc, y se considera que la ingesta no será suficiente para mantener un óptimo crecimiento y desarrollo ${ }^{(26,32)}$. La suplementación es importante y necesaria para poblaciones vulnerables en miras de reducir la prevalencia del $\mathrm{RC}{ }^{(33)}$. Sin embargo, al momento del estudio, el programa de suplementación con zinc no tenía suficiente cobertura. Deficiente alimentación junto con deficiente suplementación puede explicar la alta incidencia de infecciones en esta población.

La ingesta de zinc fue evaluada en una dieta mixta para evaluar el riesgo del bajo consumo a nivel poblacional (34). La mayoría de variantes dietéticos que se pueden encontrar en un estudio transversal fueron contemplados en el diseño metodológico ${ }^{\left({ }^{35)}\right.}$, sin embargo, la variabilidad de la dieta conforme a las épocas de cosecha es importante en esta población, dada su vulnerabilidad alimentaria, esto no fue posible tomarlo en cuenta ${ }^{(36)}$.

Comolimitaciones podemosmencionar: lasuplementación de zinc en los sujetos evaluados era irregular en el momento de la entrevista e insuficiente de acuerdo a la norma, por lo que se decidió no tomar en cuenta los suplementos en la cuantificación del consumo total del mineral. Otra limitación fue no considerar la aceptabilidad dentro del análisis; no obstante, la disponibilidad cumplía una función más importante en el consumo, dado que estas comunidades son de escasos ingresos lo que les permite acceso a ciertos alimentos. Existió la barrera del idioma chuj y mam, que requirió intérpretes. Esta tercera fuente de información puede introducir variaciones en la información registrada de difícil control. Finalmente, una limitación adicional es que los resultados aquí descritos no necesariamente pueden ser extrapolados a otras comunidades fuera de Guatemala, teniendo en cuenta las diferencias geográficas, culturales y de acceso a diferentes tipos de alimento.

Contribuciones de autoría: WC, MMV e IF han participado en la concepción del estudio, recolección y análisis de datos, redacción y aprobación de la versión final del manuscrito. JLD participó en el análisis de los datos y en la aprobación de la versión final del manuscrito. WC, MMV obtuvieron el financiamiento.

Fuentes de financiamiento: el proyecto fue financiado mayormente por los autores, con la colaboración del Ministerio de Salud Pública y Asistencia Social de Guatemala (transporte del personal de campo y gestión comunitaria).

Conflictos de interés: los autores declaran no tener conflictos de interés. 


\section{REFERENCIAS BIBLIOGRÁFICAS}

1. Joint FAO/WHO. Expert Consultation on Human Vitamin and Mineral Requirements. Vitamin and mineral requirements in human nutrition : report of a joint $\mathrm{FAO} / \mathrm{WHO}$ expert consultation [Internet]. 2nd ed. Bangkok, Thailand: WHO; 2004 [citado el 17 de junio 2017]. Disponible en: http://www.who. int/nutrition/publications/micronutrients/9241546123/en

2. Dibley MJ. Zinc. En: Bowman B, editor. Conocimientos actuales sobre nutrición [Internet]. 8va ed. Washington, DC: OPS/Instituto Internacional de Ciencias de la Vida; 2003 [citado el 17 de junio 2017]. p. 360-76. Disponible en: http://iris.paho.org/xmlui/bitstream/ handle/123456789/3150/Conocimientos\%20actuales\%20sobre\%20 nutricion\%20octava\%20edicion.pdf ?sequence $=1 \&$ is Allowed $=\mathrm{y}$

3. Krebs NF, Miller LV, Hambidge KM. Zinc deficiency in infants and children: a review of its complex and synergistic interactions. Paediatr Int Child Health. 2014;34(4):279-88. doi: 10.1179/2046905514Y.0000000151

4. De Benoist B, Darnton-Hill I, Davidsson L, Fontaine O, Hotz C. Conclusions of the Joint WHO/UNICEF/IAEA/ IZiNCG Interagency Meeting on Zinc Status Indicators. Food Nutr Bull. 2007;28(3 Suppl):S480-4. doi: $10.1177 / 156482650702835306$

5. Ministerio de Salud Pública y Asistencia Social (MSPAS). Encuesta Nacional de Salud Materno Infantil 2008/2009 (ENSMI-2008/09) [Internet]. Guatemala: MSPAS; 2011 [citado el 17 de junio 2015]. Disponible en: http://www.ine. gob.gt/sistema/uploads/2014/01/22/ LYk4A 1 kGJAO7lvfS0Aq6tezcUa9tQh35.pdf

6. Wessells KR, Brown KH. Estimating the global prevalence of zinc deficiency: results based on zinc availability in national food supplies and the prevalence of stunting. PLoS One. 2012;7(11):e50568. doi: 10.1371/journal.pone.0050568

7. Cediel G, Olivares M, Brito A, Cori H, López de Romaña D. Zinc Deficiency in Latin America and the Caribbean. Food Nutr Bull. 2015;36(2 suppl):S129-38. doi: $10.1177 / 0379572115585781$

8. Brown KH, Peerson JM, Baker SK, Hess SY. Preventive zinc supplementation among infants, preschoolers, and older prepubertal children. Food Nutr
Bull. 2009;30(1 Suppl):S12-40. doi: $10.1177 / 15648265090301 S 103$

9. Mazariegos M, Hambidge K, Krebs N, Westcott J, Lei S, Grunwald G, et al. Zinc absorption in Guatemalan schoolchildren fed normal or low-phytate maize. Am J Clin Nutr. 2006;83(1):59-64.

10. Instituto Nacional de Estadística (INE). República de Guatemala: Encuesta Nacional de Condiciones de Vida 2014 [Internet]. Guatemala: INE; 2016 [citado el 17 de junio 2017]. Disponible: https://www.ine.gob.gt/sistema/uploads/2016/02/03/bWC7f6t7aSbEI4wmuExoNR0oScpSHKyB.pdf

11. Secretaria de Seguridad Alimentaria y Nutricional (SESAN). Tercer censo nacional de talla en escolares del primer grado de primaria del sector oficial de la República de Guatemala [Internet]. Guatemala: SESAN; 2009 [citado el 15 de abril 2017]. Disponible en: https://issuu.com/webmaster.nutrinet.org/docs/resumen ejecutivo censo 08

12. Instituto Nacional de Estadística (INE). Mapas de pobreza rural en Guatemala 2011, Resumen ejecutivo [Internet]. Guatemala: INE; 2013 [citado el 27 de dic 2015]. Disponible en: http://www.ine. gob.gt/sistema/uploads/2014/01/10/ ifRRpEnf0cjUfRZGhyXD7RQjf7EQH2Er.pdf

13. Instituto Nacional de Estadística (INE). Guatemala: Estimaciones de la Población total por municipio. Periodo 2008-2020 (al 30 de junio) [Internet]. Guatemala: INE; 2008 [citado el 20 de junio 2017]. Disponible en: http://www.oj.gob. gt/estadisticaj/reportes/poblacion-total-por-municipio(1).pdf

14. Brown KH, Rivera JA, Bhutta Z, Gibson RS, King JC, Lonnerdal B, et al. International Zinc Nutrition Consultative Group (IZiNCG) technical document \#1. Assessment of the risk of zinc deficiency in populations and options for its control. Food Nutr Bull. 2004;25(1 Suppl 2):S99203.

15. Valverde C, Rodriguez M, Méndez $\mathrm{H}$. Tabulación de Punto: Metodología para Adaptar las Guías Alimentarias en el Ámbito Local (Publicación INCAP MDE/141) [Internet]. Guatemala: INCAP; 2005 [citado el 17 de junio 2017]. Disponible en: http://bvssan. incap.int/local/SAN\%20Local/Documentos\%20t\%C3\%A9cnicos/Tabulacion\%20Punto\%20para\%20adaptar\%20 guias\%20alimentarias\%20en\%20el\%20 \%C3\%A1mbito\%20local.pdf

16. Guatemala, Presidencia de la República. Acuerdo gubernativo número 75-2006, Reglamento de la Ley del Sistema Nacional de Seguridad Alimentaria y Nutricional [Internet]. Guatemala: SESAN; 2006 [citado el 17 de junio 2017]. Disponible en: http://www.sesan.gob.gt/wordpress/ wp-content/uploads/2016/02/Reglamento-de-SAN.pdf

17. Joint WHO/FAO/UNU Expert Consultation. Protein and amino acid requirements in human nutrition. World Health Organ Tech Rep Ser. 2007;(935):1-265.

18. USDA Food Composition Databases [Internet] Beltsville, MD: United States Deparment of Agriculture. Agriculture; c2015 - [citado el 17 de junio 2017]. Disponible en: http://ndb.nal.usda.gov/

19. Menchú MT, Méndez $H$, editores. Tabla de composición de alimentos de Centroamérica. 2nd ed. Guatemala: Instituto de Nutrición de Centroamérica; 2007. 128 p.

20. Thompson FE, Byers T. Dietary assessment resource manual. J Nutr. 1994;124(11 Suppl):2245S-317S.

21. Wright JD, Ervin B, Briefel RR, editores. Consensus Workshop on Dietary Assessment: Nutrition Monitoring and Tracking the year 2000 Objectives [Internet]. Maryland, United States: US Department of Health and Human Services; 1994 [citado el 26 de dic 2015]. Disponible en: http://www.cdc.gov/ nchs/data/misc/nutri94acc.pdf

22. Platel K, Srinivasan K. Bioavailability of micronutrients from plant foods: an update. Crit Rev Food Sci Nutr. 2016;56(10):1608-19. doi: 10.1080/10408398.2013.781011

23. Hotz C, Gibson RS. Traditional food-processing and preparation practices to enhance the bioavailability of micronutrients in plant-based diets. J Nutr. 2007;137(4):1097-100.

24. Dermience M, Mathieu F, Li XW, Vandevijvere S, Claus W, De Maertelaer $\mathrm{V}$, et al. Minerals and trace elements intakes and food consumption patterns of young children living in rural areas of Tibet autonomous region, P.R. China: a cross-sectional survey. Healthcare (Basel). 2017;5(1):12. doi: 10.3390/ healthcare 5010012 
25. Instituto Nacional de Estadística (INE). Encuesta Nacional Agropecuaria [Internet]. Guatemala: INE; 2014 [citado el 27 de dic 2015]. Disponible en: http:// web.maga.gob.gt/download/ena-2013. pdf

26. Brown KH, Peerson JM, Rivera J, Allen LH. Effect of supplemental zinc on the growth and serum zinc concentrations of prepubertal children: a meta-analysis of randomized controlled trials. Am J Clin Nutr. 2002;75(6):1062-71.

27. Müller $O$, Becher $H$, van Zweeden $A B$, Ye Y, Diallo DA, Konate AT, et al. Effect of zinc supplementation on malaria and other causes of morbidity in west African children: randomised double blind placebo controlled trial. BMJ. 2001;322(7302):1567.

28. Roohani N, Hurrell R, Kelishadi R, Schulin R. Zinc and its importance for human health: an integrative review. J Res Med Sci. 2013;18(2):144-57.

29. Bhutta ZA, Black RE, Brown KH, Gardner JM, Gore S, Hidayat A, et al. Prevention of diarrhea and pneumonia by zinc supplementation in children in developing countries: pooled analysis of randomized controlled trials. Zinc Investigators' Collaborative Group. J Pediatr. 1999;135(6):689-97.

30. Biblioteca electrónica de documentación científica sobre medidas nutricionales (eLENA) [Internet]. Genova: OMS; c2011-2017. Administración de suplementos de zinc para mejorar los resultados terapéuticos en niños diagnosticados de infección respiratoria: abril del 2011 [citado el 26 de dic 2016]; [una página]. Disponible en: http:// www.who.int/elena/titles/bbc/zinc_ pneumonia_children/es/

31. Brown KH. Diarrhea and malnutrition. J Nutr. 2003;133(1):328S-32S.

32. Owino V, Ahmed T, Freemark M, Kelly P, Loy A, Manary M, et al. Environmental enteric dysfunction and growth failure/ stunting in global child health. Pediatrics. 2016;138(6): e20160641. doi: 10.1542/ peds.2016-0641

33. Sánchez J, Villada OA, Rojas ML, Montoya L, Díaz A, Vargas C, et al. Efecto del zinc aminoquelado y el sulfato de zinc en la incidencia de la infección respiratoria y la diarrea en niños preescolares de centros infantiles. Biomédica. 2013;34(1):79-91. 10.7705/biomedica.v34i1.1581

34. Gibson RS, Yeudall F, Drost N, Mtitimuni B, Cullinan T. Dietary interventions to prevent zinc deficiency. Am J Clin Nutr. 1998;68(2):484S-87S.

35. Bell LK, Golley RK, Magarey AM. Short tools to assess young children's dietary intake: a systematic review focusing on application to dietary index research. J Obes. 2013;2013:709626. doi: $10.1155 / 2013 / 709626$

36. Consejo Nacional de Seguridad Alimentaria u Nacional (CONASAN). Plan estratégico de seguridad alimentaria y nutricional (PESAN) 2012-2016. Guatemala: CONASAN; 2011.

Correspondencia: Michele Monroy-Valle Dirección: Vista Hermosa III, Campus Central, zona 16, edificio L, oficina 308. Nueva Guatemala de la Asunción, Guatemala. Teléfono: (+502) 24262626, (+502) 57002466.

Correo electrónico:mmmonroy@gmail.com

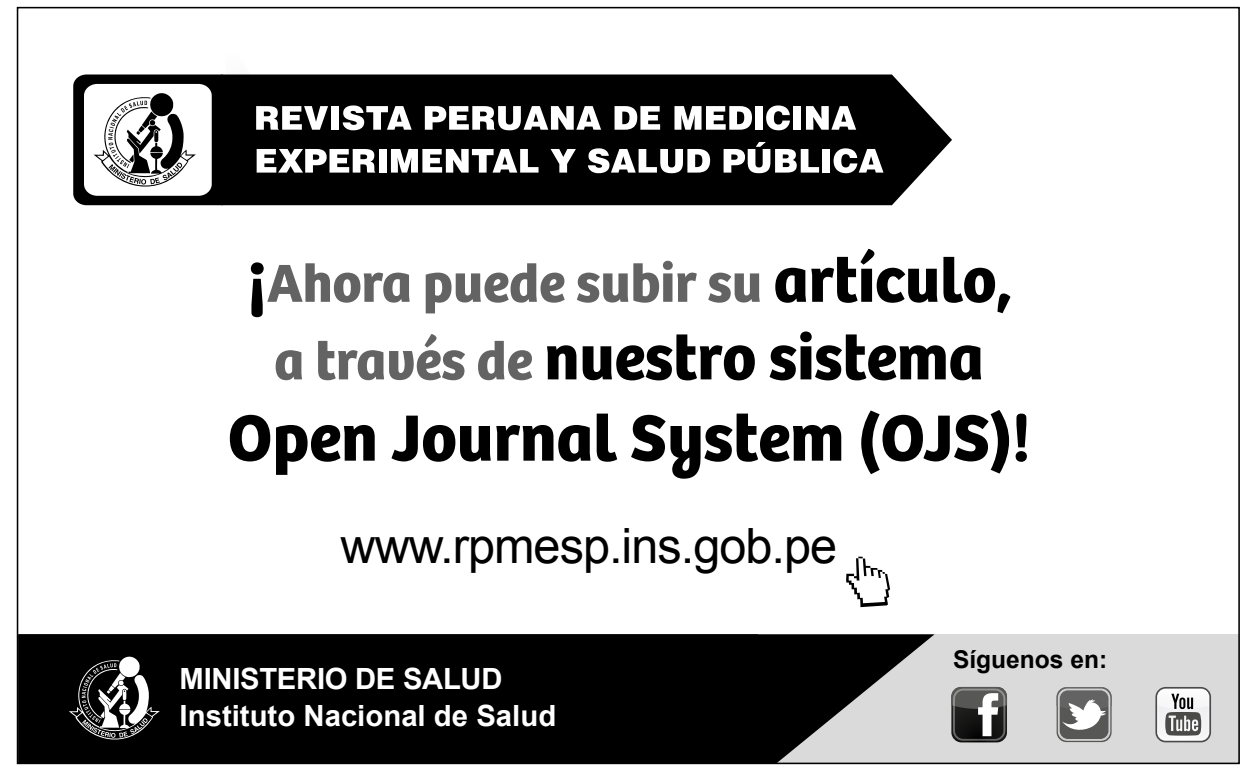

